\title{
Size effect measurement and characterization in nanoindentation test
}

\author{
Yueguang Wei, ${ }^{\text {a) }}$ Xuezheng Wang, and Manhong Zhao \\ LNM, Institute of Mechanics, Chinese Academy of Sciences, \\ Beijing 100080, People's Republic of China
}

(Received 16 June 2003; accepted 3 October 2003)

\begin{abstract}
Nanoindentation test at scale of hundreds of nanometers has shown that measured hardness increases strongly with decreasing indent depth, which is frequently referred to as the size effect. Usually, the size effect is displayed in the hardness-depth curves. In this study, the size effect is characterized in both the load-displacement curves and the hardness-depth curves. The experimental measurements were performed for single-crystal copper specimen and for surface-nanocrystallized Al-alloy specimen. Moreover, the size effect was characterized using the dislocation density theory. To investigate effects of some environmental factors, such as the effect of surface roughness and the effect of indenter tip curvature, the specimen surface profile and the indentation imprint profile for single-crystal copper specimen were scanned and measured using the atomic force microscopy technique. Furthermore, the size effect was characterized and analyzed when the effect of the specimen surface roughness was considered.
\end{abstract}

\section{INTRODUCTION}

Indentation test is an important and effective experimental method and has extensively been used to estimate the plastic properties of solids undergoing plastic deformation. Through indentation test, the material parameters, such as yield strength, strain hardening exponent, and Young's modulus, are estimated. Recently, with advancement in experimental technique and measuring precision, it is possible to carry out the indentation experiment at scale levels of tens to hundreds of nanometers. Such a small-scale indentation test is referred to as the nanoindentation test (or microindentation test). In the nanoindentation test, an important phenomenon, sizedependent indentation result, has been attained for metal materials $;{ }^{1-14}$ that is, the measured hardness may double or even triple the conventional hardness as indent size (or depth) decreases to one-fifth of a micrometer. The effect is often referred to as the size effect. In addition, at the micrometer scale when material microstructured size is comparable to the indent depth, the size-dependent hardness results mentioned above should be influenced additionally by the geometrical size. ${ }^{10}$ Regarding the sensitive zone size of the size effect, for the typical metals, such as $\mathrm{Cu}, \mathrm{Ag}$, and $\mathrm{Al}^{2,7,9,11}$ within one or one-half micrometer of the indent depth, the hardness is sensitive

\footnotetext{
a) Address all correspondence to this author.
} e-mail: ywei@Lnm.imech.ac.cn to indent depth. However, for some uniquely high modulus metals, such as $\mathrm{W}$ and $\mathrm{Ir}^{8,13}$ the size effect sensitive zone size is much larger, that is, within about tens of micrometers or even 100 micrometers the hardness is still sensitive to depth. The trends of both size effect and geometrical effect are at odds with the size-independence implied by the conventional elastic-plastic (CEP) theory.

To predict the size effect phenomenon in nanoindentation test, several versions of strain gradient plasticity theories were developed. ${ }^{3,4,9,10,14-17}$ These investigators took the strain gradient effect as the dominant factor in the size effect sensitive zone. Simultaneously, a dislocation density model was used to study the size effect in nanoindentation test ${ }^{1,13,16}$ and to set up the relationship between the strain gradient and the geometrically necessary dislocation density. ${ }^{16,17}$

Usually, the indentation size effect was described in the hardness-displacement curves ${ }^{1-14}$ Because the most direct results of the nanoindentation test are the loaddisplacement curves, it must be important and interesting to characterize the indentation size effect in the loaddisplacement curves. This will be the main intention in the current research. In nanoindentation test, the factors affecting the experimental results from experimental environments, ${ }^{18}$ such as the specimen surface roughness, the indenter tip curvature, the pile-up or sink-in deformations, exist inevitably. Therefore, it is also important to investigate these effects on the experimental results. In the current study, the load-displacement (contact depth) 
curves for single-crystal copper and for surfacenanocrystallized Al-alloy material are measured experimentally, and the size effects are characterized in the load-displacement curves within the submicrometer region using the dislocation density theory. In addition, as usual the size effects are also characterized in the hardness-displacement curves. To explore the effects of the factors mentioned above, such as the specimen surface roughness, indenter tip curvature, the elastic recovery, and the pile-up (sink-in) deformation, the profiles of the specimen surface and the imprint shape are scanned, measured, and analyzed by using the atomic force microscopy (AFM) technique for the single-crystal copper specimen. Finally, as a byproduct of the current research, the connection between the indentation hardness and the mathematical error estimate of the loaddisplacement relations will be presented.

\section{EXPERIMENTAL}

First, the specimen surfaces of the single-crystal $\mathrm{Cu}$ and the surface-nanocrystallized Al-alloy material were ground and polished with number 600-1200 sand sheets smoothly and carefully. Second, one surface to be indented was polished on the polishing machine using the finer media, such as diamond paste with 0.1-nm average particle size. After the polishing process, chemical methods were used to remove the remainders on the surface and eliminate residual stress within the surface layer. Nanoindentation experiments were conducted on the test instrument MTS-Nanoindenter II using the Berkovich (three-sided pyramid) indenter with about a 40-nm tip curvature radius. Displacements and loads were measured with a resolution of $0.7 \mathrm{~nm}$ and $0.3 \mu \mathrm{N}$, respectively. The continuous stiffness measurement using the Oliver and Pharr ${ }^{19}$ model was adopted.

To investigate some environmental effects, such as the effects of the surface roughness and indenter tip curvature, the measurements of profiles of the specimen surface and the indent imprints were performed by using the AFM technique for the single-crystal copper specimens. Experiments were performed in the Institute of Metal Materials, Chinese Academy of Sciences (Shenyang, P.R.C.), and the AFM measurements were performed in the Physical Laboratory, the Physical Department, Tsinghua University (Beijing, P.R.C.).

\section{RESULTS}

\section{A. Single-crystal copper specimen}

Adopting the continuous stiffness measurement method for randomly selected test points on the specimen surface (110), load-displacement curves were measured, as shown in Fig. 1. Simultaneously, hardnessdisplacement curves were also measured and are shown

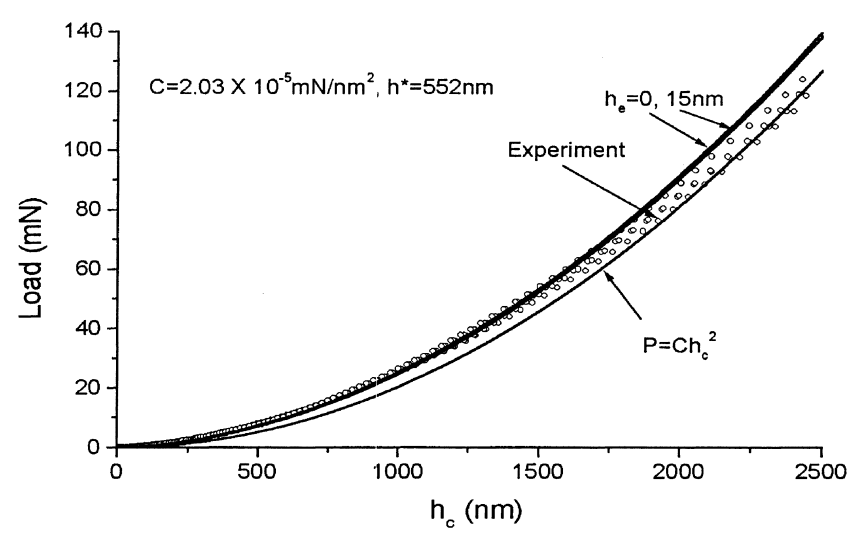

(a)

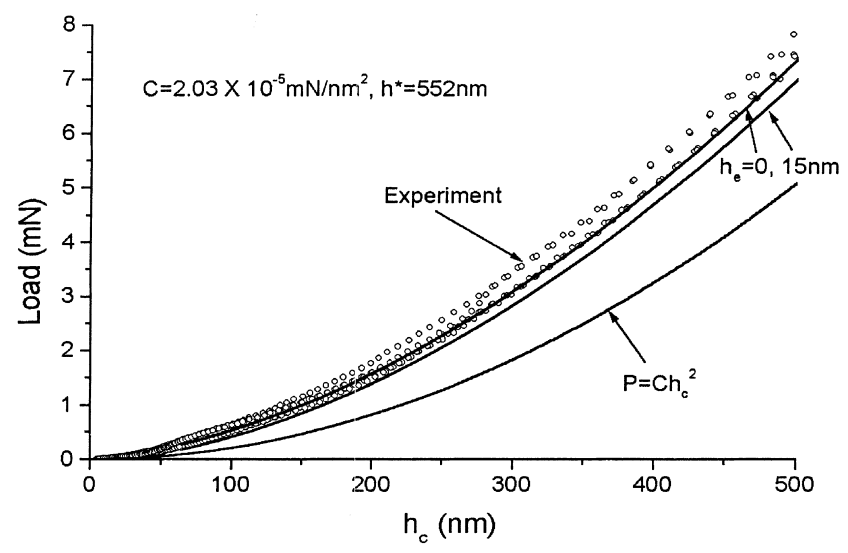

(b)

FIG. 1. Load-displacement curves for single-crystal $\mathrm{Cu}$ shown in (a) large-scale coordinates and in (b) small-scale coordinates. Conventional elastic-plastic theory result and the dislocation density method results considering the specimen surface roughness effects are also shown.

in Fig. 2. To investigate the effect of elastic recovery deformation, the loading-unloading curves were measured for five loading stages from $0.75 \mathrm{mN}$, corresponding to $100-\mathrm{nm}$ depth, to a final load of $124 \mathrm{mN}$, corresponding to 2000-nm depth, as shown in Fig. 3. In the figures, the horizontal coordinate, displacement $h_{\mathrm{c}}$, corresponds to the contact depth of indentation. The experimental results, load-displacement curves, and loadingunloading curves, are relatively smooth. However, the corresponding hardness curves are not smooth, especially within the submicrometer region of the contact depth. For comparison, the CEP theory simulated curve, a parabola, which will be discussed in detail in Sec. IV, is plotted in the figures. From Figs. 1(a) and 3(a), the loaddisplacement curves seem to be well-simulated with the CEP theory solution; however, through detailed investigation there is a big difference between them within the submicrometer region. This can be found in Fig. 1(b). Figure 1(b) is the same figure as Fig. 1(a) except for adopting different scales of coordinates. Obviously, in the small scale the difference between experimental data and the con- 


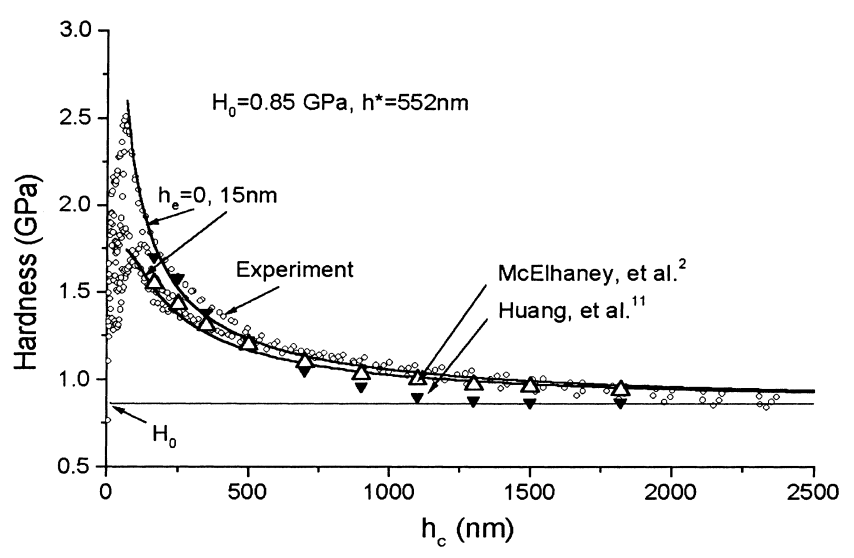

(a)

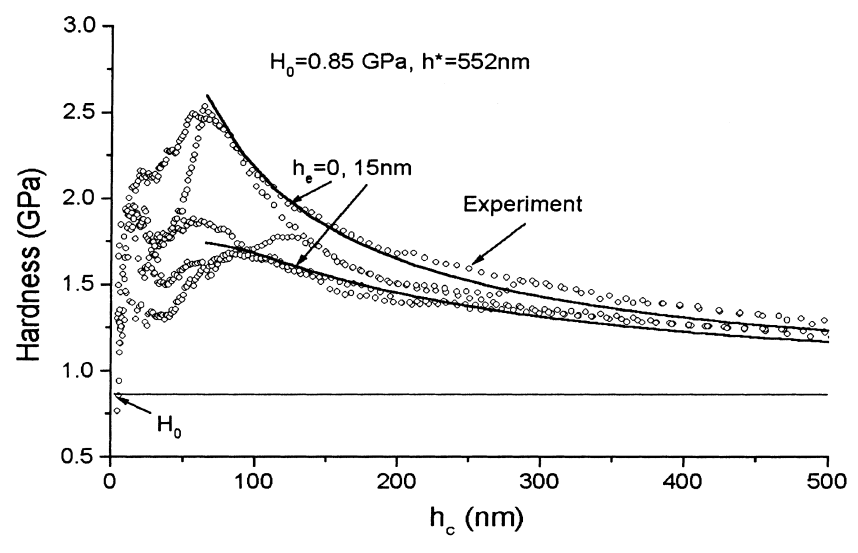

(b)

FIG. 2. Hardness-displacement curves for single-crystal $\mathrm{Cu}$ shown in (a) large-scale coordinates and in (b) small-scale coordinates. Conventional elastic-plastic theory result and the dislocation density method results considering the specimen surface roughness effects are also shown.

ventional theory result is considerably big. This difference is described clearly in hardness curves, as shown in Fig. 2. The hardness curve from the parabolic law shown in Fig. 1 corresponds to a horizontal straight line with a constant value $H_{0}$, as shown in Fig. 2. The difference between the experimental curves and the CEP theory simulation is called size effect in nanoindentation test. In Fig. 2(a), the experimental results for materials of the single-crystal copper $^{11}$ and strain-hardened copper ${ }^{2}$ are also shown for comparison. The effectiveness of the current experimental results is confirmed. From Figs. 1 and 3, the experimental load-displacement curves are relatively smooth, and the differences between curves are small, so that the effect of the specimen surface roughness on the curves seems negligible. However, from Fig. 2, for hardness curves, this effect is magnified, especially within the indent depth region of $300 \mathrm{~nm}$. This effect will be discussed later in Sec. IV. C.

Besides the effect of the specimen surface roughness, the indenter tip curvature effect on the experimental results is also displayed. From Figs. 2(a) and 2(b), within

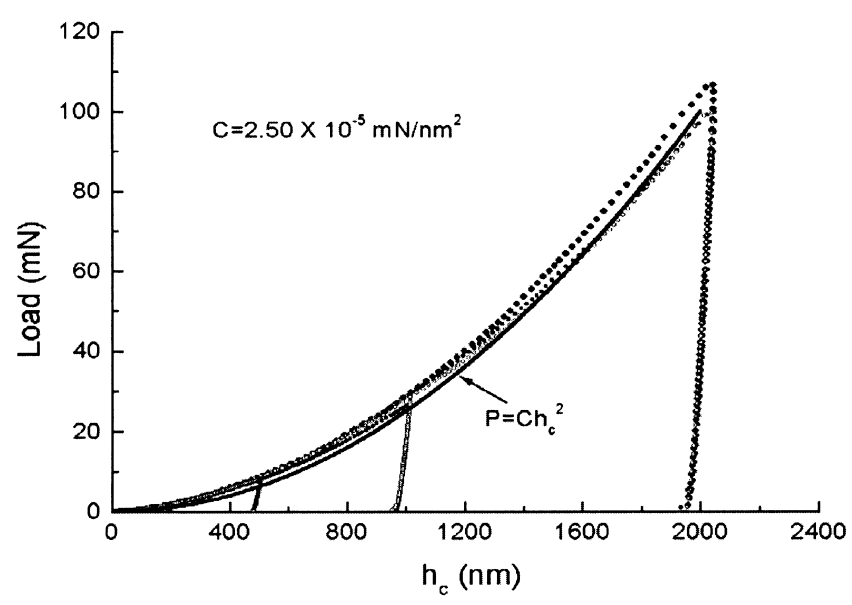

(a)

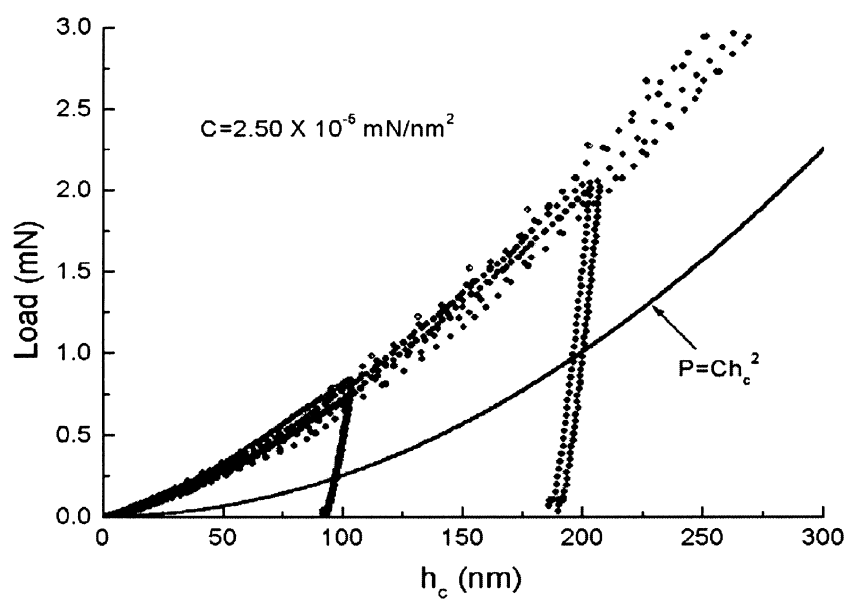

(b)

FIG. 3. Loading-unloading curves for single-crystal $\mathrm{Cu}$ specimen shown in (a) large-scale coordinates and in (b) small-scale coordinates.

the depth region of about $60 \mathrm{~nm}$, the hardness value decreases as depth decreases. The feature is consistent with the analysis of Xue et al. ${ }^{12}$ for the effect of indenter tip curvature.

\section{B. Surface-nanocrystallized Al-alloy material}

To investigate the size effects further, the nanoindentation experiment for surface-nanocrystallized Al-alloy material was made on the cross-section points corresponding to the crystal grain size around $20 \mathrm{~nm}$. The surface-nanocrystallized Al-alloy material is an improved modern composite made by using a nanocrystalline technique. ${ }^{20}$ The distribution of crystal-grain sizes is in a gradient manner along the thickness, which is several nanometers on the nanocrystalline surface and several tens of micrometers inside the material far away from the nanocrystalline surface. Near the material surface, the nanocrystalline layer thickness is about $80 \mu \mathrm{m}$. Figures 4 and 5 show the load-displacement curves and the hardness-displacement curves, respectively. Figures 4(b) 


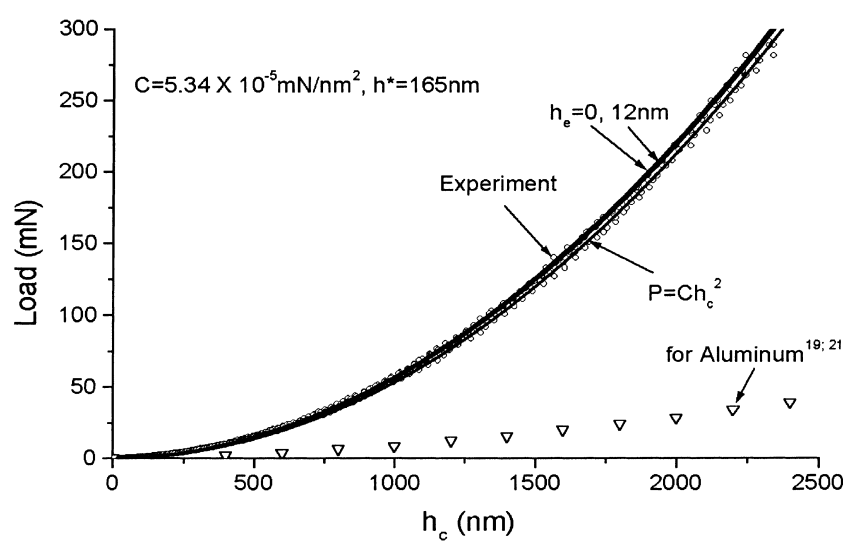

(a)

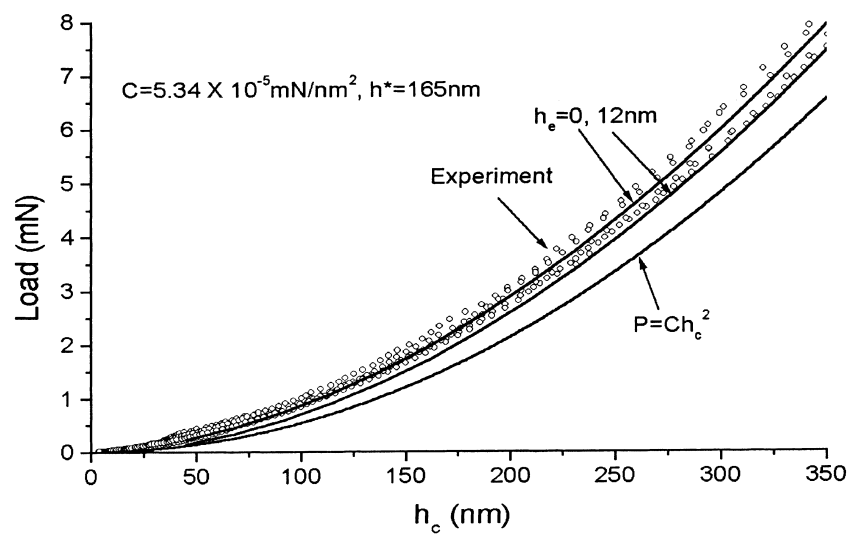

(b)

FIG. 4. Load-displacement curves for surface-nanocrystallized Alalloy specimen shown in (a) large-scale coordinates and in (b) smallscale coordinates. Conventional elastic-plastic theory result and the dislocation density method results considering the specimen surface roughness effects are also shown.

and 5(b) correspond to the replotted figures of Figs. 4(a) and 5(a), respectively, with $350 \mathrm{~nm}$ as the maximum horizontal coordinate. For comparison, Fig. 4(a) also shows the experimental load-displacement result for conventional aluminum. ${ }^{19,21}$ Through comparison, one finds that the nanocrystallized Al-alloy material has the much higher hardness value than that of the conventional aluminum. From Fig. 4(a), in large-scale coordinates, load-displacement curves seem to be well simulated by the CEP theory result (parabolic relation), which will be discussed in detail in Sec. IV. However, from Fig. 4(b), in small-scale coordinates the difference between the experimental curves and the parabolic curve is relatively large. The hardness-displacement curves are plotted in Figs. 5(a) and 5(b).

Comparing the results in Figs. 2 and 5, one can observe that the size-effect sensitive zone size for the surface-nanocrystallized Al-alloy material seems smaller than that for the single-crystal copper material. The hardness curve of the surface-nanocrystallized Al-alloy material is higher than that of the single-crystal copper material.

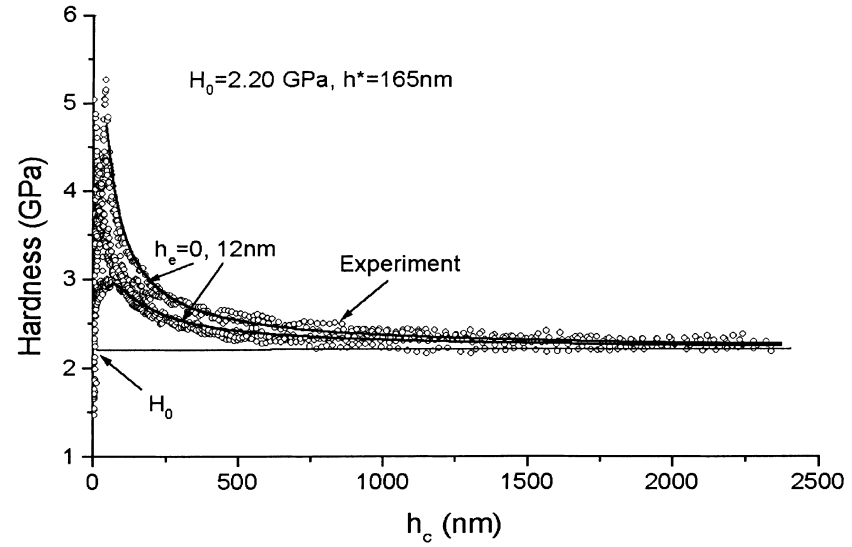

(a)

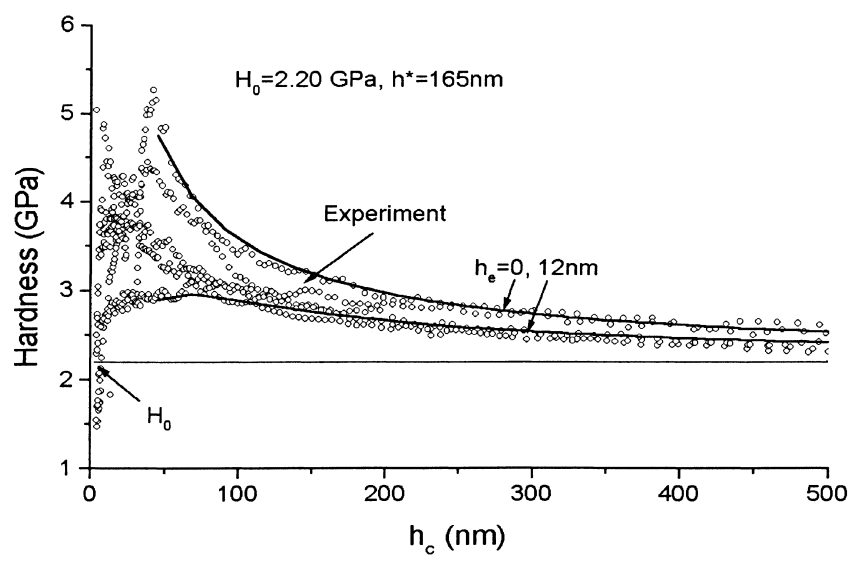

(b)

FIG. 5. Hardness-displacement curves for surface-nanocrystallized Al-alloy specimen shown in (a) large-scale coordinates and in (b) small-scale coordinates. Conventional elastic-plastic theory result and the dislocation density method results considering the specimen surface roughness effects are also shown.

\section{AFM measurements for the single-crystal copper specimens}

From experimental hardness curves shown in Figs. 2 and 5, the experimental results have undergone considerable effects from surface roughness and indenter tip curvature within a small region of tens of nanometers. To investigate the roughness effects, a nanoscale measurement for copper specimens was performed using AFM technique. Before the experiments, the surface profile was scanned and measured. After the experiments, the profiles of the indentation imprints were measured. Figure 6 shows the specimen surface profile measured by using the AFM technique. From Fig. 6, as expected, a zig-zag surface shape in nanoscale is displayed. Figures 6(a)-6(d) show the surface profile scanned along four different straight lines: a horizontal, a vertical, and two inclined lines, respectively. Locations of each line on the specimen surface were selected arbitrarily before scanning. From the profile figures, the root mean square roughness, ${ }^{22,23}$ 

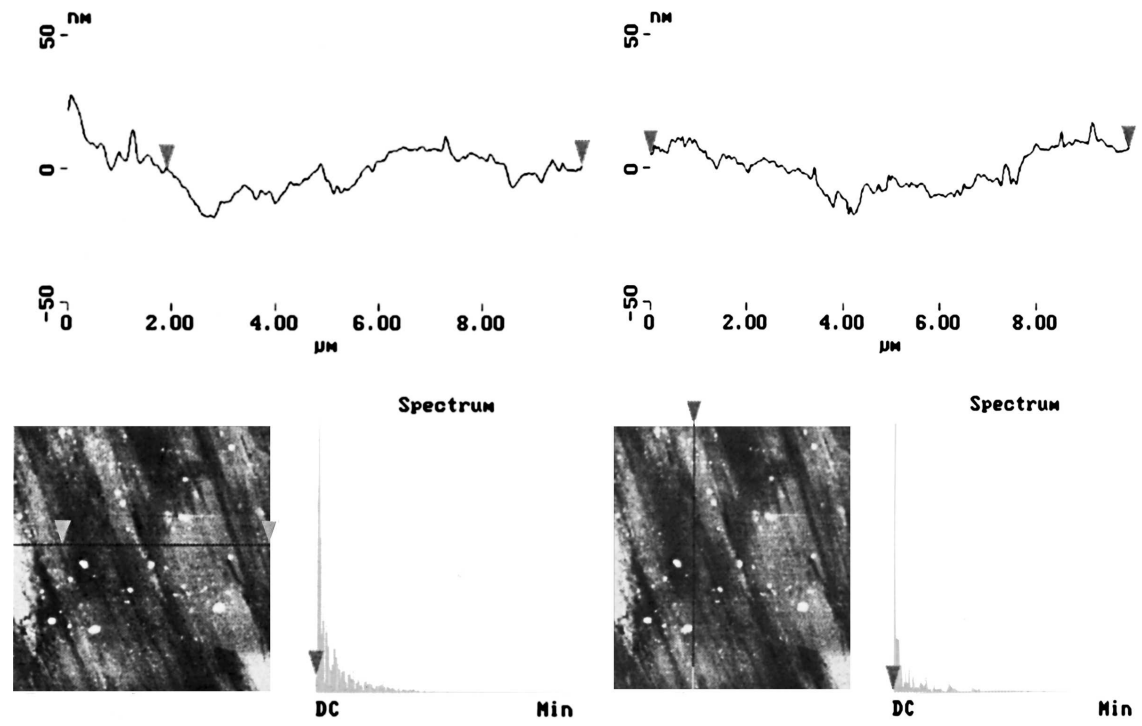

(a)

(b)

ho $^{\mathrm{nm}}$

$\operatorname{Bn}^{-n M}$
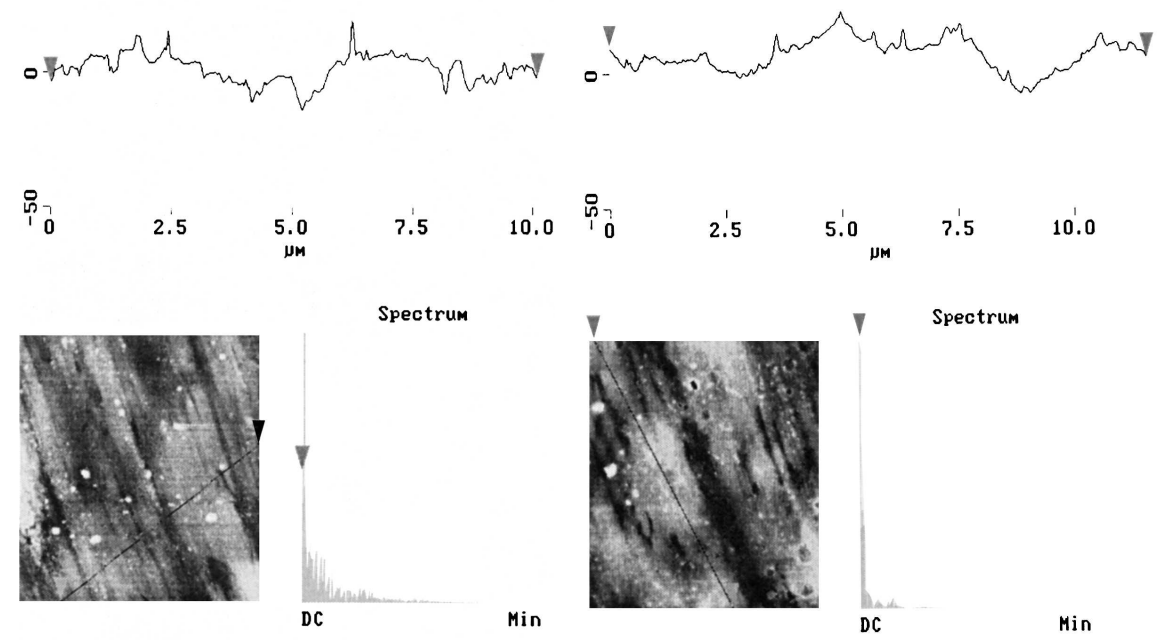

$\begin{array}{lll}5.0 & 7.5 & 10.0\end{array}$

(c)

(d)

FIG. 6. Photos and curves of surface profiles of the single-crystal copper scanned along four lines on the specimen surface using the AFM technique. The surface roughness can be measured based on the curves.

$$
h_{\mathrm{r}}=\sqrt{\left(z_{1}^{2}+z_{2}^{2}+\ldots+z_{\bar{N}}^{2}\right) / \bar{N}}
$$

an average roughness value for a surface, was measured, where $\left(z_{1}, z_{2}, \ldots z_{N}\right)$ are the vertical coordinates of surface points relative to an average horizontal plane and $\bar{N}$ is the number of the measured points. In the current measurement, each line included 50 measured points, and total measured points were $\bar{N}$. The result of the root mean square roughness obtained as $h_{\mathrm{r}} \approx 5 \mathrm{~nm}$. Moreover, to investigate the effects of the indenter tip curvature, the profiles of indentation imprint for several depth cases were measured after the experiments. First, the complete pictures of the imprints for two indent depths were taken by using the AFM technique, as shown in Fig. 7. The AFM photos were taken for two depths of the imprints, $500 \mathrm{~nm}$ and $1000 \mathrm{~nm}$, respectively. In these photos, to display the imprint profiles clearly, AFM inverse imaging technique was adopted. Indent depth is described with brightness (i.e., the deeper, the brighter). The curvature radii of the imprint tips are displayed in Figs. 7(a) and 7(b). The effects of the indenter tip curvature can be observed from the profile photos of the imprints. Furthermore, the profiles of the imprints along the outlines crossing the apex, one side, and the central line of the triangular cross section of the imprint were measured for three indent depth cases, $h_{c}=1000 \mathrm{~nm}, 500 \mathrm{~nm}$, and 


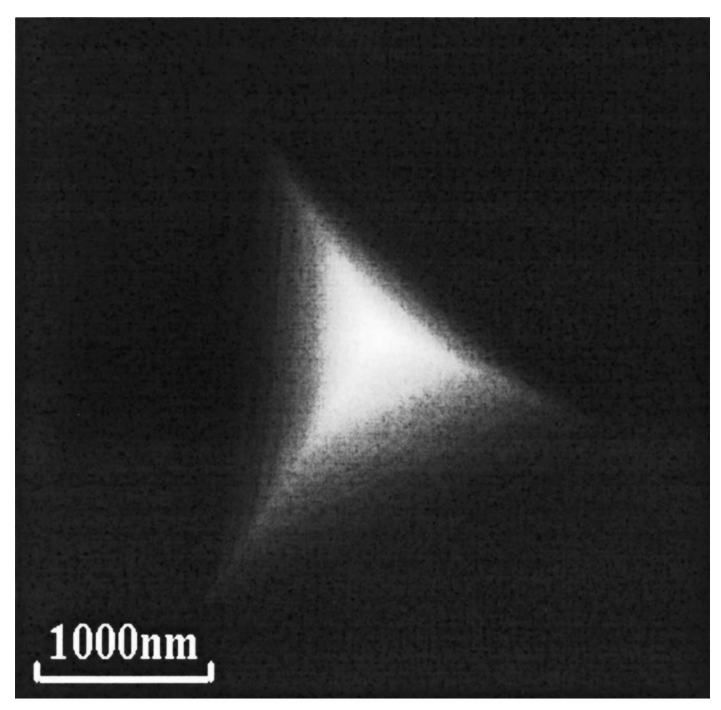

(a) $h_{c}=500 \mathrm{~nm}$

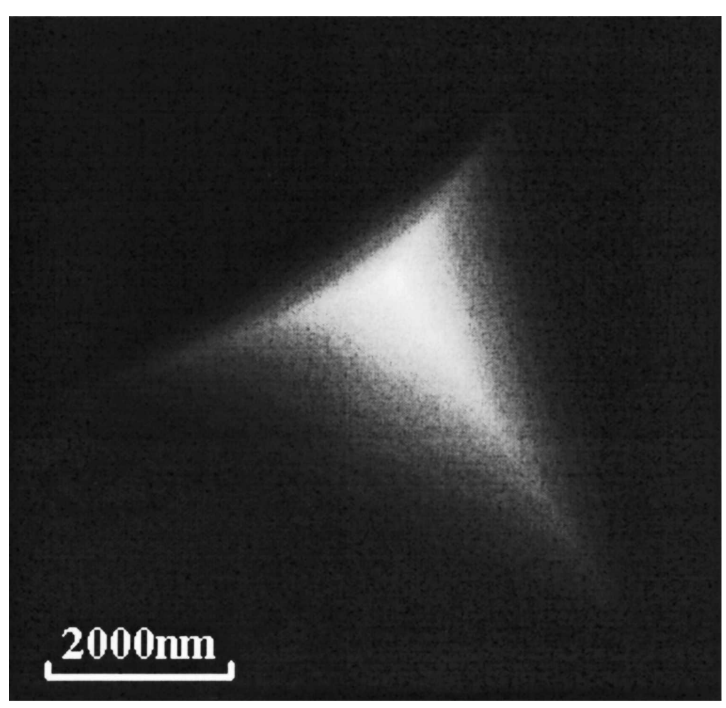

(b) $h_{c}=1000 \mathrm{~nm}$

FIG. 7. The complete pictures of the indentation imprints for two indent depth cases using the AFM inversion technique. Indent depth is described with brightness.

200 nm, shown in Figs. 8(a)-8(c), respectively. From Figs. 7 and 8 , the pyramidal shape of the imprints seems to be well kept after unloading, and the effects of the indenter tip curvature seem not to play too big of a role in the indentation test results. The pile-up and sink-in phenomena are not observed obviously. On the other hand, from loading-unloading curves shown in Fig. 3, the unloading recovery deformation is not too big, even for small indent depth. Moreover, from Figs. 7 and 8, the indenter tip curvature radius observed by means of the imprints is not obvious. Actually, the effects of the indenter tip curvature on the hardness curves, if any, should be within a small indent depth region at the same order as

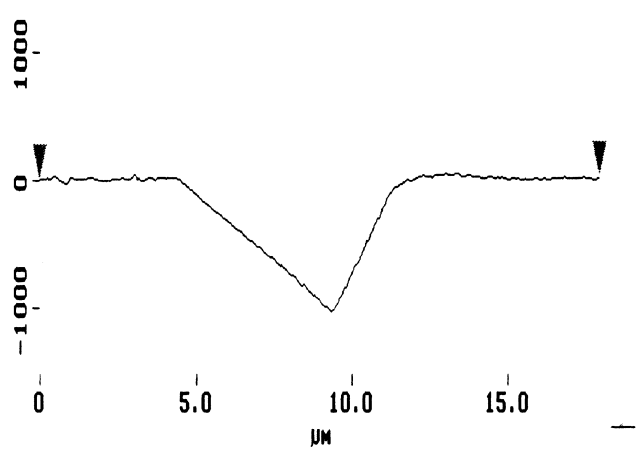

(a) $h_{c}=1000 \mathrm{~nm}$
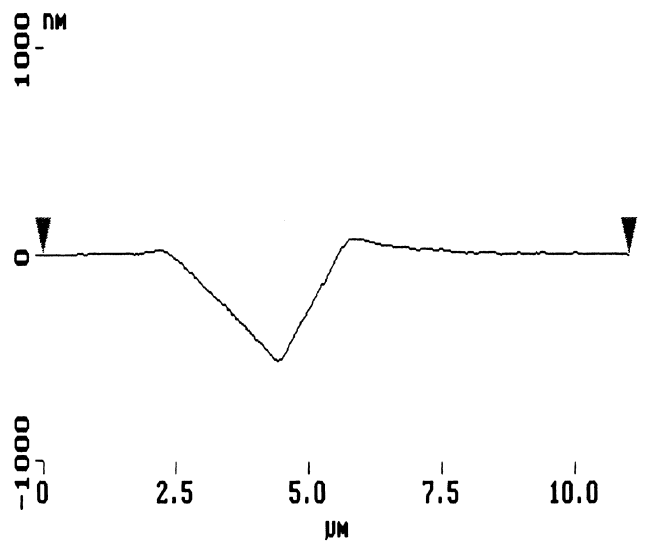

(b) $h_{c}=500 \mathrm{~nm}$

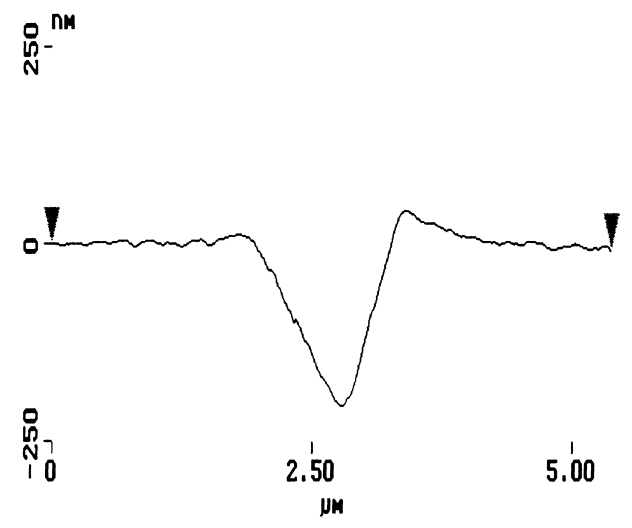

(c) $h_{c}=200 \mathrm{~nm}$

FIG. 8. Pictures of profiles of the indentation imprints for three indent depths using the AFM technique. Scanning is along the apex and the one side of the imprint.

the indenter tip curvature radius. For the pyramidal indenter in the MTS-Nanoindenter II, there exists a small curvature radius at the indenter tip, around $40 \mathrm{~nm}$ from manual. Conceivably, during indentation experiment, at start a small spherical surface contacts with the specimen 
surface. According to the researches of Swadener et al. ${ }^{13}$ and Xue et al. ${ }^{12}$ for a spherical indenter case, the hardness increases as indent depth increases. From current experimental results shown in Figs. 2(b) and 5(b), it seems reasonable to propose that within the region $h_{c}<$ $40 \mathrm{~nm}$, the indenter tip contacts the specimen surface with a spherical surface; the trends of experimental results within the small region seem consistent with the result trends obtained by Swadener et al. ${ }^{13}$ and Xue et al. ${ }^{12}$ The discrepancy of hardness curves within the small region will be interpreted as the effects of the specimen surface roughness in Sec. IV. C below.

\section{CHARACTERIZATION OF SIZE EFFECT}

Size effect describes the different behaviors of material manifested in different length scales from macroscale to microscale. There are several kinds of theoretical methods for characterizing the size effect. The dislocation density theory method and the strain gradient theory method are two of them. According to previous researches of our group, ${ }^{9}$ the results of the size effect modeling based on both methods were very similar with each other for properly selecting the modeling parameters included in both theories. In the following analysis, the dislocation density theory method will be used. Moreover, the effect of the specimen surface roughness on the nanoindentation test result will be discussed.

\section{A. Conventional elastic-plastic theory result}

The size-effect phenomenon cannot be described by adopting the CEP theory. According to dimensional analysis, ${ }^{24}$ the indentation load can be expressed in the form

$$
P=h_{c}^{2} C\left(E / \sigma_{\mathrm{Y}}, \nu, N, \beta\right)=h_{c}^{2} \sigma_{\mathrm{Y}} f\left(E / \sigma_{\mathrm{Y}}, \nu, N, \beta\right),
$$

where $h_{c}$ is the indent contact depth, $E$ is material Young's modulus, $v$ is Poisson's ratio, $\sigma_{\mathrm{Y}}$ is yield strength, $N$ is material strain hardening exponent for a power-law hardening material, $\beta$ is the pyramidal indenter tip angle, and for the instrument Nanoindenter II, $\beta$. The horizontal cross-sectional area of the contact surface is $A \approx 24.5 h_{c}^{2}$; from Eq. (1), hardness can be expressed as:

$$
\begin{aligned}
H & =(1 / 24.5) C\left(E / \sigma_{\mathrm{Y}}, \nu, N, \beta\right) \\
& =\left(\sigma_{Y} / 24.5\right) f\left(E / \sigma_{\mathrm{Y}}, \nu, N, \beta\right) .
\end{aligned}
$$

For a weakly hardening metal material, $0<N<0.2$, the hardness is approximately equal to three times the yield strength (i.e., $H \approx 3 \sigma_{\mathrm{Y}}$ ). ${ }^{25}$

From Eq. (1), for the CEP theory, the loaddisplacement curve is a parabolic curve, and the hardness curve [Eq. (2)] is independent of the indent depth.
The predicted results from Eqs. (1) and (2) are shown in Fig. 1-5 in solid lines. The value of $C$ depends on the material parameters and the indenter angle and can be computed through using the finite element method ${ }^{9,25}$ to simulate the nanoindentation experiment and can also be directly obtained through using the parabolic curve [Eq. (1)] to simulate the nanoindentation experimental curve. The obtained $C$ values using the above two methods are very close to each other. ${ }^{9}$ In the current study, the determination of $C$ is through letting the value of the CEP theory result be equal to the value of the experimental load-displacement curves at the end point (i.e., at the maximum value of indent depth).

\section{B. Dislocation density theory method}

The nanoindentation test for metals belongs to the case of the nonuniform plastic strain problem. From the dislocation density theory, the dislocation density is also nonuniform. In this case, the dislocation density can be separated into two parts: the statistically stored dislocation density and the geometrically necessary dislocation density. ${ }^{26,27}$ It depends not only on the exerted load, but also on the microstructure geometrical parameters. ${ }^{26,27}$ The geometrically necessary dislocation density depends on the geometrical parameters. Nix and $\mathrm{Gao}^{1}$ obtained a simple and an approximate relation of the total dislocation density relation for nanoindentation test problem, $\rho_{\mathrm{T}}$ $=B\left(1+h^{*} / h_{\mathrm{c}}\right)$, and the total dislocation density depends on the indent depth $h_{\mathrm{c}}$. Putting together the related relations, such as the Taylor model relation, von Mises flow theory of plasticity, Tabor factor relation, and the above dislocation density relation, we have

$$
\begin{aligned}
\tau & =\alpha \mu b \sqrt{\rho_{\mathrm{T}}}, \\
\rho_{\mathrm{T}} & =B\left(1+h^{*} / h_{c}\right), \sigma=\sqrt{3} \tau, \\
H & =3 \sigma,
\end{aligned}
$$

where $\tau$ and $\sigma$ are the shear flow stress and von Mises effective flow stress, respectively, $\mu$ is shear modulus, $b$ is Burger's vector, $\alpha$ is a geometrical constant and its value is about 0.3 , and $B$ and $h^{*}$ are constants to be determined in the nanoindentation test. $B$ is statistically stored dislocation density and $h^{*}$ is a characteristic length, characterizing a varying strength of the geometrically necessary dislocation density. For $h_{c}<h^{*}$, the geometrically necessary dislocation density prevails; otherwise, the statistically stored dislocation density prevails. From Eq. (3), Nix and Gao derived out ${ }^{1}$

$$
H=H_{0} \sqrt{1+h^{*} / h_{c}},
$$

where

$$
H_{0}=3 \sqrt{3} \alpha \mu b \sqrt{B}
$$

$H_{0}$ is the macroscale hardness without size effects and should be equal to $H$ in Eq. (2) for using the CEP theory. Therefore, from Eq. (4), and by using Eqs.(1) and (2), 
one can readily derive out the load-displacement relation as follows:

$$
P=\sqrt{1+\frac{h^{*}}{h_{c}}} h_{c}^{2} C\left(\frac{E}{\sigma_{\mathrm{Y}}}, \nu, N, \beta\right) .
$$

In the hardness relation (4) and the load-displacement relation (6), the characteristic parameter $h^{*}$ can be determined through using the hardness relation (4) to simulate experimental curve. The simulated results by using (4) and (6) are shown in Figs. 1 and 2 and Figs. 4 and 5, corresponding to $h_{\mathrm{e}}=0$ (without roughness effect). From Figs. 1 and 2 and Figs. 4 and 5, obviously, the trends of the load-displacement curves and the hardnessdepth curves can be described by using the dislocation density theory, except for a small region with the size of several tens of nanometers. From Figs. 1(a) and 4(a), the simulated results of the load-displacement curves using the CEP theory $\left(P=C h_{c}^{2}\right)$ seem to compare well with the experimental curves assessed from the length scale of several micrometers. However, the comparisons are poorly assessed from the submicrometer scale as shown in Figs. 1(b) and 4(b). From the hardness-displacement curves, the CEP theory simulations always compare poorly to the experimental curves from Figs. 2 and 5 . The simulated results using the dislocation density method compare well with the experimental curves for both the load-displacement curves and the hardnessdisplacement curves, except for a small region of indent depth.

\section{Surface roughness effects}

The discrepancy of hardness curves within the small indent depth region [Figs. 2(b) and 5(b)] can be interpreted as the specimen surface roughness effects. Following the method of Weiss ${ }^{22}$ and Bobji et al., ${ }^{23}$ the effect of specimen surface roughness on the hardness curve can be considered with a revision on the results without roughness effect. This implies that the measurement error for contact depth due to the specimen surface roughness is deducted or eliminated through adopting an effective indent depth to replace the original depth. Referring to Eq. (4), considering the effect of the surface roughness, the hardness-depth relation can be expressed as: ${ }^{22,23}$

$$
H=\left(1-\frac{h_{\mathrm{e}}}{h_{c}}\right)^{2} \sqrt{1+\frac{h^{*}}{h_{c}-h_{\mathrm{e}}}} H_{0},
$$

where

$$
h_{\mathrm{e}}=k h_{\mathrm{r}},
$$

$h_{\mathrm{r}}$ is the root mean square roughness of the surface $\mathrm{e}^{22,23}$ as described above in Sec. III. C. From investigation by Bobji et al., ${ }^{23}$ the coefficient $k$ is about 3. From Eq. (7), one can obtain the load-displacement relation considering the effects of the surface roughness as

$$
P=\left(1-\frac{h_{\mathrm{e}}}{h_{c}}\right)^{2} \sqrt{1+\frac{h^{*}}{h_{c}-h_{\mathrm{e}}}} h_{c}^{2} C\left(\frac{E}{\sigma_{\mathrm{Y}}}, \nu, N, \beta\right) .
$$

From the current AFM measurement for the copper specimen surface in Fig. 6, we have $h_{r} \approx 5 \mathrm{~nm}$ (as presented in Sec. III.C), and thus $h_{\mathrm{e}} \approx 5 \mathrm{~nm}$. Figures 1 and 2 also show the load-displacement curves and hardnessdisplacement curves considering the surface roughness effect. From Figs. 1(a) and 1(b), the roughness effect on the load-displacement curves is small. However, the roughness effect on the hardness-depth curves is obvious within the small depth region of $300 \mathrm{~nm}$. The effects of the surface roughness on the load-displacement curves and the hardness-depth curves for the surfacenanocrystallized Al-alloy specimen are given in Figs. 4 and 5, where take $h_{\mathrm{e}}$ to be $12 \mathrm{~nm}$. Obviously, the roughness effect on the hardness-depth curves is considerably large within a small region of the indent depth.

\section{ERROR ESTIMATE}

For the load-displacement relations, the comparison of the CEP theory simulated result with the experimental result is performed under the mathematical concepts absolute error and relative error. A connection between error measurement of both results and the indent hardness is presented.

\section{A. The absolute error}

Let $e_{\mathrm{a}}^{\mathrm{L}}$ and $e_{\mathrm{a}}^{\mathrm{H}}$ be the absolute errors of the load-and hardness-displacement curves between the experimental result and the CEP theory simulation result, respectively. Thus,

$$
\begin{aligned}
& e_{\mathrm{a}}^{\mathrm{L}}\left(h_{c}\right)=\left|P_{\exp }-C h_{c}^{2}\right|=\left(\frac{H_{\exp }}{H_{0}}-1\right) C h_{c}^{2}, \\
& \text { for } h_{\mathrm{i}}<h_{c}<h_{\max }, \\
& e_{\mathrm{a}}^{\mathrm{H}}\left(h_{c}\right)=\left|H_{\exp }-H_{0}\right|=\left(\frac{H_{\exp }}{H_{0}}-1\right) H_{0}, \\
& \text { for } h_{\mathrm{i}}<h_{c}<h_{\max } .
\end{aligned}
$$

$P_{\text {exp }}$ and $H_{\text {exp }}$ are the experimental load and hardness. $C h_{c}^{2}$ and $H_{0}$ are the load and hardness simulated using the CEP theory. $\left(h_{\mathrm{i}}, h_{\max }\right.$ is the effective range of the experimental curves; from current experimental results, $h_{\mathrm{i}} \approx$ $70 \mathrm{~nm}$, and $h_{\max } \approx 2400 \mathrm{~nm}$. According to an agreement in Figs. 1-5, we have $e_{\mathrm{a}}^{\mathrm{L}}\left(h_{\text {max }}\right)=0$ and $e_{\mathrm{a}}^{\mathrm{H}}\left(h_{\text {max }}\right)=0$, the conditions to determine $C$ and $H_{0}$. From Figs. 2(a) and 2(b) and Figs. 5(a) and 5(b), $e_{\mathrm{a}}^{\mathrm{H}}\left(h_{c}\right)$ increases monotonically as $h_{c}$ decreases from $h_{\max }$ to $h_{\mathrm{i}}$. At $h_{c}=h_{\mathrm{i}}, e_{\mathrm{a}}^{\mathrm{H}} \approx$ 
1.4 GPa from both Figs. 2 and 5. From Figs. 1(a) and 1(b), Figs. 3(a) and 3(b), and Figs. 4(a) and 4(b), the variation of $e_{\mathrm{a}}^{\mathrm{L}}\left(h_{\mathrm{c}}\right)$ is complicated as $h_{c}$ decreases. The maximum value of $e_{\mathrm{a}}^{\mathrm{L}}$ is about $6 \mathrm{mN}$ at $h_{c} \approx 1256 \mathrm{~nm}$ from Fig. 1(a) and is about $3 \mathrm{mN}$ at about $700 \mathrm{~nm}$ of indent depth. Because the absolute error is dimensiondependent, it is not suitable for a scale problem.

\section{B. The relative error}

Let $e_{\mathrm{r}}^{\mathrm{L}}$ and $e_{\mathrm{r}}^{\mathrm{H}}$ be the relative errors of the load- and hardness-displacement curves between the experimental result and the CEP theory simulation result, respectively. Thus

$$
\begin{aligned}
e_{\mathrm{r}}^{\mathrm{L}}\left(h_{c}\right) & =\frac{\left|P_{\exp }-C h_{c}^{2}\right|}{C h_{c}^{2}}=\left|\frac{H_{\exp }}{H_{0}}-1\right| \\
& =\frac{H_{\exp }}{H_{0}}-1, \quad \text { for } h_{\mathrm{i}}<h_{c}<h_{\max }, \\
e_{\mathrm{r}}^{\mathrm{H}}\left(h_{c}\right)= & \frac{\left|H_{\exp }-H_{0}\right|}{H_{0}}=\frac{H_{\exp }}{H_{0}}-1, \\
& \text { for } h_{\mathrm{i}}<h_{c}<h_{\max } .
\end{aligned}
$$

From the last two formulas, the relative error functions have a correlation with the material hardness-depth curves. According to the agreement for determining $H_{0}$, we have $e_{\mathrm{r}}^{\mathrm{L}}\left(h_{\max }\right)=e_{\mathrm{r}}^{\mathrm{H}}\left(h_{\max }\right)=0$. The relative errors increase as the indent depth decreases monotonically. At $h_{\mathrm{i}} \approx 70 \mathrm{~nm}, e_{\mathrm{r}}^{\mathrm{L}}=e_{\mathrm{r}}^{\mathrm{H}}$ is about 2.0 from Fig. 2 for singlecrystal copper specimen and is about 1.4 from Fig. 5 for the surface-nanocrystallized Al-alloy material specimen.

From the above error analyses for difference of the load-displacement relations between the experiment and CEP theory modeling, the relative error is a dimensionless quantity and has the obvious physical meaning, that is, it is equivalent to the material hardness ratio. Therefore, the relative error is a suitable quantity for assessing the size effect or the consistence of the CEP theory result with the experimental result in both large scale and small scale.

\section{CONCLUSIONS}

A nanoindentation experimental result, such as the load-displacement curve, covers multiple scales from microscale to macroscale. At macroscale, one can use the CEP theory to simulate the macroscale experimental result. However, when one uses the CEP theory to simulate the microscale experimental result, a big relative error or a big hardness difference between the experimental result and the modeling result can occur. The current researches show that the depth-dependence of the dislocation density may be the size effect dominated factor in nanoindentation test.
The size effects have been characterized by using the dislocation density method in the current research. From simulations for the experimental processes of the copper specimen and of the surface-nanocrystallized Al-alloy material specimen, we note that the dominant zone sizes of the geometrically necessary dislocation density for both kinds of specimens, characterized by $h^{*}$, are different from each other: $h^{*}=552 \mathrm{~nm}$ for the single-crystal copper specimen and $h^{*}=165 \mathrm{~nm}$ for the surfacenanocrystallized Al-alloy specimen, from Figs. 1 and 2. It is worth pointing out that the geometrically necessary dislocation density can be directly connected to the strain gradient of solids. ${ }^{26,27}$ Based on the concept of the geometrically necessary dislocation density, Gao et al. ${ }^{16}$ and Huang et al. ${ }^{17}$ further developed a strain gradient plasticity theory. As mentioned above in Sec. IV, both dislocation density theory and strain gradient theory have a certain connection with each other. Therefore, $h^{*}$ also characterizes the strain gradient dominated zone size. From this point, there exists a strain gradient dominated zone around the imprint tip. The zone size of the singlecrystal copper specimen is larger than that of the surfacenanocrystallized Al-alloy specimen.

The effects of the specimen surface roughness and the indenter tip curvature have been investigated by using AFM measurement and the revised method of Weiss ${ }^{22}$ and Bobji and Biswas. ${ }^{23}$ Both effects only play a role in a small region with tens of nanometers.

\section{ACKNOWLEDGMENTS}

This work was supported by the National Natural Science Foundation of China through Grant 19925211 and jointly supported by the Chinese Academy of Sciences through "Bai Ren Plan." The assistance from Dr. Wu Xiaolei in preparing surface-nanocrystallized Al-alloy specimen is sincerely acknowledged.

\section{REFERENCES}

1. W.D. Nix and H. Gao, J. Mech. Phys. Solids 46, 411 (1998).

2. K.W. McElhaney, J.J. Vlassak, and W.D. Nix, J. Mater. Res. 13, 1300 (1998).

3. M. Begley and J.W. Hutchinson, J. Mech. Phys. Solids 46, 1029 (1998).

4. J.Y. Shu and N.A. Fleck, Int. J. Solids Struct. 35, 1363 (1998).

5. W.J. Poole, M.F. Ashby, and N.A. Fleck, Scr. Metall. Mater. 34, 559 (1996).

6. M. Atkinson, J. Mater. Res. 10, 2908 (1995).

7. Q. Ma and D.R. Clarke, J. Mater. Res. 10, 853 (1995).

8. N.A. Stelmashenko, M.G. Walls, L.M. Brown, and Y.V. Milman, Acta Metall. Mater. 41, 2855 (1993).

9. Y. Wei, X. Wang, X. Wu, and Y. Bai, Science in China (Series A) 44, 74 (2001).

10. Y. Wei, X. Wang, M. Zhao, C.M. Cheng, and Y.L. Bai, Acta Mech. Sin. 19, 59 (2003).

11. Y. Huang, Z. Xue, H. Gao, W.D. Nix, and Z.C. Xia, J. Mater. Res. 15, 1786 (2000). 
12. Z. Xue, Y. Huang, K.C. Hwang, and M. Li, J. Eng. Mater. Technol. 124, 371 (2002).

13. J.G. Swadener, E.P. George, and G.M. Pharr, J. Mech. Phys. Solids 50, 681 (2002).

14. Y. Wei and J.W. Hutchinson, J. Mech. Phys. Solids 51, 2037 (2003, in press).

15. N.A. Fleck and J.W. Hutchinson, Adv. Appl. Mech. 33, 295 (1997).

16. H. Gao, Y. Huang, W.D. Nix, and J.W. Hutchinson, J. Mech. Phys. Solids 47, 1239 (1999).

17. Y. Huang, H. Gao, W.D. Nix, and J.W. Hutchinson, J. Mech. Phys. Solids 48, 99 (2000).

18. A. Iost and R. Bigot, J. Mater. Sci. 31, 3573 (1996).
19. W.C. Oliver and G.M. Pharr, J. Mater. Res. 7, 1564 (1992).

20. K. Lu and J. Lu, J. Mater. Sci. Technol. 15, 193 (1999).

21. Y.T. Cheng and C.M. Cheng, J. Mater. Res. 13, 1059 (1998).

22. H.J. Weiss, Phys. Status Solidi A129, 167 (1992).

23. M.S. Bobji and S.K. Biswas, J. Mater. Res. 14, 2259 (1999).

24. Y.T. Cheng and C.M. Cheng, Int. J. Solids Struct. 36, 1231 (1999).

25. M.C. Shaw, in Mechanical Behavior of Materials, edited by F.A. McClintock and A.S. Argon (Addison-Wesley, Reading, MA, 1966), p. 443.

26. M.F. Ashby, Philos. Mag. 21, 399 (1970).

27. N.A. Fleck, G.M. Muller, M.F. Ashby, and J.W. Hutchinson, Acta Metall. Mater. 42, 475 (1994). 